\title{
Dexmetomidine promotes the activity of breast cancer cells through miR-199a/HIF-1 $\alpha$ axis
}

\author{
Gang Wen, Naixing Xin \\ Department of Anesthesiology, Maternal and Child Health Hospital of Hubei Province, Wuhan, China \\ Contributions: (I) Conception and design: N Xin; (II) Administrative support: G Wen; (III) Provision of study materials or patients: G Wen; \\ (IV) Collection and assembly of data: Both authors; (V) Data analysis and interpretation: G Wen; (VI) Manuscript writing: Both authors; (VII) Final \\ approval of manuscript: Both authors. \\ Correspondence to: Naixing Xin. Department of Anesthesiology, Maternal and Child Health Hospital of Hubei Province, No. 745 Wuluo Road, \\ Hongshan District, Wuhan 430070, China. Email: znnezn2021@163.com.
}

\begin{abstract}
Background: Breast cancer, as one of the most common malignant tumors in women, is still a great threat to women all over the world. Dexmetomidine (DMED) is a highly selective $\alpha 2$-adrenergic receptor agonist, which has attracted much attention in recent years. This study aimed to clarify the potential mechanism of DMED in regulating the activity of breast cancer cells.

Methods: Breast cancer cell lines MCF-7 and MDA-MB-231 were treated with DMED. The levels of miR-199a and HIF-1 $\alpha$ mRNA were detected using quantitative real-time polymerase chain reaction (QRTPCR); the 3-(4,5-dimethylthiazol-2-yl)-2,5-diphenyl-2H-tetrazolium bromide (MTT) and transwell assays were applied to monitor the activity of breast cancer cells; the apoptosis of breast cancer cells was detected using the caspase- 3 activity assay and flow cytometry; binding of miR-199a and HIF-1 $\alpha$ was assessed using double luciferase reporter gene assay, and western blot was employed to monitor the level of HIF-1 $\alpha$ in cells.

Results: The cytotoxicity and apoptosis of MCF-7 and MDA-MB-231 cells was inhibited by DMED. It also downregulated the expression of miR-199a in breast cancer cells and enhanced the downregulation of miR-199a to promote the activity of breast cancer cells and inhibit apoptosis. Also, miR-199a targeted HIF-1 $\alpha$. Further functional experiments confirmed that DMED promoted the progression of breast cancer through the miR-199a/HIF-1 $\alpha$ axis.
\end{abstract}

Conclusions: DMED promotes the activity of breast cancer cells through miR-199a/HIF-1 $\alpha$ axis. This can provide some reference for DMED in the clinical treatment of breast cancer.

Keywords: Dexmetomidine (DMED); breast cancer; miR-199a; HIF-1 $\alpha$

Submitted Jul 27, 2021. Accepted for publication Oct 02, 2021.

doi: $10.21037 /$ tcr-21-1937

View this article at: https://dx.doi.org/10.21037/tcr-21-1937

\section{Introduction}

The incidence of breast cancer is second only to cervical cancer in women, and with the gradual increase in incidence in recent years, breast cancer has become one of the foremost malignant tumors threatening the life and health of urban women $(1,2)$. At present, clinicians mainly determine whether breast cancer is present according to the patient's clinical signs, imaging manifestations, and related tumor markers. Early diagnosis is the key to improve the cure rate of breast cancer, but there is a lack of obvious clinical features during the early stages of breast cancer. In recent years, although important progress has been made in the treatment of breast cancer in the areas of surgery, radiotherapy, chemotherapy, and endocrine and targeted therapies, the prognosis of patients has not significantly improved. The molecular mechanisms of the occurrence and development of breast cancer need to be assessed further $(3,4)$. Studies have confirmed that drugs play an important role in the progression of breast cancer. For 
example, flavonoids such as quercetin, as dietary substances of great concern to ordinary people, have potential as alternative or supplementary drugs for breast cancer (5). On the other hand, hyperin plays a role as an anticancer drug by activating the Bax-caspase- 3 axis and inhibiting the NF$\kappa \mathrm{B}$ signal pathway through reactive oxygen species (ROS)related apoptosis (6). Therefore, in-depth elucidation of the mechanisms of drugs in the treatment of breast cancer has important clinical significance.

Dexmetomidine (DMED) is a highly selective $\alpha 2-$ adrenergic receptor agonist, which is mainly used as a sedative and analgesic in clinical practice. There is evidence that DMED can exert anti-apoptosis and protective functions on cells and organs $(7,8)$. Studies have proven that DMED promotes metastasis in rodent models of breast, lung, and colon cancer (9). Also, Zhang et al. have shown that DMED inhibits the progression of esophageal cancer through the miR-143-3p/EPS8 axis (10). On the other hand, DMED promotes the migration of breast cancer cells through exocrine TMPRSS2 secretion mediated by Rab11 (11). Similarly, DMED could promote the proliferation, migration and invasion of breast cancer cells through the activation of a2B-adrenoceptor/ERK signaling (12). Whereas, the role of DMED in regulating the miRNA-mRNA axis in the malignant phenotype of breast cancer is not clear.

MicroRNA (miRNA) is an evolutionarily conserved noncoding RNA containing 19-22 nucleotides. It not only plays an important role in regulating the normal development of organisms but also participates in the regulation of the pathological processes of many types of malignant tumors (13). The role of miRNAs in the occurrence and development of breast cancer has been reported (14). For example, previous studies have shown that dual targeting of miR-124-3p and ABCC4 promotes the sensitivity of breast cancer cells to doxorubicin (15). Also, MiR-199a has been shown to inhibit the activity of breast cancer cells (16). Wu et al. have confirmed that DMED prevents oxidative damage to PC12 cells by regulating miR-199a/HIF-1 $\alpha$ (17). At the same time, DMED attenuates neuronal injury induced by cerebral ischemia-reperfusion (IR) by regulating miR199a (18). However, the role of DMED in regulating miR199a in breast cancer is unknown. Moreover, Cao et al. have confirmed that HIF-1 $\alpha$ can promote the activity of breast cancer cells (19). This shows that HIF- $1 \alpha$ plays a cancerpromoting role in breast cancer. In view of the current state of research, in this study, MCF-7 and MDA-MB-231 breast cancer cell lines were treated with different concentrations of DMED to explore the role of DMED in promoting the activity of breast cancer cells through the miR-199a/HIF$1 \alpha$ axis. The results showed that DMED could inhibit the downregulation of miR-199a and promote the activity of breast cancer cells. Further experiments confirmed that DMED promoted the progression of breast cancer by regulating the miR-199a/HIF-1 $\alpha$ axis.

We present the following article in accordance with the MDAR reporting checklist (available at https://dx.doi. org/10.21037/tcr-21-1937).

\section{Methods}

\section{Cell culture}

Human breast cancer cell lines (MCF-7 and MDAMB-231) were purchased from ATCC (Rockville, IN, USA). The cells were cultured in RPMI-1640 complete medium (GIBCO, Carlsbad, CA, USA) containing $10 \%$ fetal bovine serum (FBS, HyClone, Logan, UT, USA) and $1 \%$ penicillin/streptomycin, cultured in an incubator at $37{ }^{\circ} \mathrm{C}$ and $5 \% \mathrm{CO}_{2}$ saturated humidity; the medium was changed every $2-3$ days. When the cells were close to fusion, they were digested and passaged with $0.25 \%$ trypsin (20). DMED purchased from Chen Xin Pharmaceutical Co., Ltd. DMED at different concentrations $(0,1,10$ and $100 \mathrm{~nm})$ were added to the medium of breast cancer cells. After 24 hours of incubation, the medium was renewed.

\section{Cell transfection}

MCF-7 and MDA-MB-231 cells in logarithmic growth phase were inoculated on 6-well plates with a cell density of $5 \times 10^{5} /$ well. When the cells had grown to $50 \%$ to $60 \%$, the miR-199a mimics or miR-199a inhibitors of $100 \mathrm{nmol} /$ $\mathrm{L}$ and the control group were transfected into MCF7 and MDA-MB-231 cells according to the instructions of lipid Lipofectamine 2000 transfection reagents (ThermoFisherScience, Waltham, MA, USA). The cells were collected after incubation for 48 hours, and the transfection efficiency was detected by quantitative realtime polymerase chain reaction (QRT-PCR).

\section{QRT-PCR}

After the total RNA of cells in each group was extracted with Trizol reagent and the concentration was detected, it was reverse transcribed into cDNA according to the 
operation instructions of Takara kit, and amplified and detected. Reaction conditions: pre denaturation at $95{ }^{\circ} \mathrm{C}$ for $30 \mathrm{~s}$, denaturation at $95^{\circ} \mathrm{C}$ for $5 \mathrm{~s}$, annealing/extension at $60{ }^{\circ} \mathrm{C}$ for $30 \mathrm{~s}$, a total of 40 cycles. The relative expression of target gene was calculated by $2^{-\Delta \Delta \mathrm{CT}}, \Delta \mathrm{CT}=$ target gene - $\beta$-actin, $\Delta \Delta=\Delta \mathrm{CT}$ experiment $-\Delta \mathrm{CT}$ control. As the internal references for miR-199a and HIF-1 $\alpha$ mRNA, U6 and $\beta$-actin were employed, respectively. The primers were synthesized by Shanghai Shenggong Biology Co., Ltd. (Shanghai, China).

\section{Detection of cell activity using 3-(4,5-dimethylthiazol-2- yl)-2,5-diphenyl-2H-tetrazolium bromide (MTT) assay}

Cells in logarithmic growth phase were taken to prepare a $1 \times 10^{3} / \mathrm{mL}$ single cell suspension, $100 \mathrm{~L}$ per well, inoculated on a 96 well plate, $37{ }^{\circ} \mathrm{C}, 5 \% \mathrm{CO}_{2}$, incubated in incubator, and after 24 hours of conventional culture, serum-free medium was used under aseptic conditions. Different reagents were added to the negative control group and interference group for cell transfection. The culture medium of each well was discarded and $20 \mu \mathrm{L}$ MTT solution $(5 \mathrm{mg} / \mathrm{mL}$, MedChem Express, Monmouth Junction, NJ, USA) was added to each well, and the cells were cultured for 4 hours. The culture medium in the hole was removed, $150 \mu \mathrm{L}$ dimethyl sulfoxide was added to each well, and it was put on the shaker and vibrated at low speed for 10 minutes to dissolve the crystal completely. Absorbance value was measured at $490 \mathrm{~nm}$ of each well with the microplate meter. The average value of six wells was taken as the result, and the absorbance value was used to express the cell proliferation. All experiments were performed 3 times in each group (21).

\section{Caspase-3 activity detection}

Steps were as follows: collection of cells, washing with phosphates buffered saline (PBS), extraction of cell lysate, protein quantification, sodium dodecyl (lauryl) sulfate-polyacrylamide gel (SDS)-PAGE electrophoresis, nitrocellulose membrane or polyvinylidene difluoride (PVDF) membrane transfer, $5 \%$ skimmed milk powder blocking for 2 hours, $4{ }^{\circ} \mathrm{C}$ overnight, caspase- 3 poly-antibody or monoclonal antibody reaction at room temperature for 2 hours, and tris-buffered saline (TBS) (containing $0.05 \%$ Tween $20 \mathrm{TBS}$ ) washed 3 times, $5-10 \mathrm{~min} /$ times. Horseradish peroxidase (HRP)-labeled sheep anti-mouse immunoglobulin (IgG) or alkaline phosphatase (AP)-labeled sheep anti-mouse IgG reacted at room temperature, followed by washing 3 times with TBS and polysorbate 20, 5-10 min each time, ECL development or nitro blue tetrazolium/5bromo-4-chloro-indolyl-phosphate (NBT/BCIP) staining.

\section{Detection of cell migration ability by transwell}

The transwell cell movement test was used to detect cell migration and invasion. In all, $2 \times 10^{4}$ cells/well were added to the upper transwell chamber, and $600 \mu \mathrm{L}$ culture medium containing $20 \%$ FBS was added to the lower chamber and cultured at $37^{\circ} \mathrm{C}$. After 12 hours, the upper chamber cells were removed, fixed using $4 \%$ paraformaldehyde, stained with $0.1 \%$ crystal violet, dried, photographed and counted. All experiments were performed 3 times.

\section{Detection of apoptosis by flow cytometry}

Apoptosis was detected using annexin V-fluorescein isothiocyanate (FITC) double staining. Twenty-four hours after transfection, the cells were digested using trypsin, collected and inoculated in a 6-well plate. The cell density was adjusted to $2 \times 10^{6}$ cells per well. The cells were cultured for 24 hours, and the supernatant was discarded. After precooling the PBS washing twice, binding buffer was used to suspend cells. Human $5 \mu \mathrm{L}$ annexin V-FITC and $5 \mu \mathrm{L}$ propidium iodide (PI) were added to the cell suspension, and then incubated at room temperature for 15 minutes. Flow cytometry was used to detect the apoptosis rate within 1 hour, and the operation was performed according to the instructions of the kit. All experiments were performed 3 times.

\section{Double luciferase reporter gene experiment}

293 T cells were digested with trypsin and re-suspended in a cell medium containing $10 \%$ FBS after centrifugation and inoculated on a 48 -well plate at a density of $4 \times 10^{4}$ cells per well. After 24 hours of culture (when the cell density was $40 \%$ to $50 \%$ ), the corresponding miR-199a expression vector (20 nmol/L), HIF-1 $\alpha$ 3'UTR reporter vector (80 ng) and pRL-TK (expressing sea kidney luciferase protein as internal reference, $40 \mathrm{ng}$ ) were co-transfected into $293 \mathrm{~T}$ cells using calcium phosphate transfection. Forty-eight hours after transfection, the cells were lysed with DualLuciferase Reporter Assay (Promega, Madison, WI, USA), and the luciferase activity was determined using a multi- 
function enzyme labeling instrument.

\section{Western blot}

After the cells were treated, the culture medium was abandoned, protein lysate (Roche, Basel, Switzerland) was added, and all proteins were separated. A total of $50 \mathrm{~g}$ of protein was added to $12 \%$ polyacrylamide gel and subjected to $100 \mathrm{~V}$ electrophoresis for 2 hours. It was then electrically transferred to a PVDF membrane. After sealing with 5\% skimmed milk powder at room temperature for 1 hour, the membrane was washed 3 times with TBST, each time for 10 min and incubated with an antibody (concentration $1: 1,000)$ overnight at $4{ }^{\circ} \mathrm{C}$. After washing the membrane with TBST, the membrane was incubated at room temperature with HRP labeled anti-rabbit second antibody (concentration $1 / 2,000)$ for $1 \mathrm{~h}$. Then, the membrane was washed 3 times with TBST, 10 min each time. Finally, western blot special reagent (Invitrogen, Waltham, MA, USA) was used for color imaging, and ImageJ was used to analyze the gray value of each protein. All experiments were performed 3 times.

\section{Statistical analysis}

The data were analyzed using SPSS 17.0 statistical software (SPSS Inc., Chicago, IL, USA). Measurement data were expressed using mean \pm standard deviation $(\bar{x} \pm \mathrm{s})$. Multiple factors were compared using one-way analysis of variance, and independent sample $t$-tests were used to compare two groups. If $\mathrm{P}<0.05$, the difference between the two groups was considered significant.

\section{Results}

\section{DMED induced cytotoxicity and apoptosis in breast cancer}

The inhibitory effect of DMED on the growth of breast cancer cells and normal breast epithelial cells was detected using MTT assay. DMED promoted the growth of MCF7 and MDA-MB-231 cells in a dose-dependent manner (Figure 1A). Flow cytometry analysis showed that the number of apoptotic MCF-7 and MDA-MB-231 cells decreased significantly after treatment with DMED (Figure $1 B$ ). The effect of DMED on apoptosis of MCF-7 and MDAMB-231 cells was dose-dependent. The activation of caspase is important in the process of apoptosis (22). To determine whether DMED can change the activity of caspase in breast cancer cells, the activity of caspase- 3 was determined. As shown in Figure 1C, DMED significantly increased the activity of caspase-3 in MCF-7 and MDA-MB-231 cells but had no effect on the activity of caspase- 3 in mammary epithelial cells.

\section{DMED down-regulated miR-199a expression}

Then, we explored the effect of DMED on the level of miR-199a in breast cancer cells. QRT-PCR results showed that DMED downregulated the level of miR-199a in MCF7 and MDA-MB-231 cells in a dose-dependent manner $(\mathrm{P}<0.05$, Figure $2 A, 2 B)$. Futhermore, DMED upregulated the level of HIF- $1 \alpha$ in MCF-7 and MDA-MB-231 cells in a dose-dependent manner $(\mathrm{P}<0.05$, Figure $2 C, 2 D)$.

\section{DMED increased the expression of miR-199a, downregulated the activity of breast cancer cells, and inbibited apoptosis}

To investigate the effect of DMED on the activity and apoptosis of breast cancer cells, MiR-199a inhibitor was used to transfect MCF-7 and MDA-MB-231 cells. Results showed that miR-199a inhibitor was successfully transfected $(\mathrm{P}<0.05$, Figure $3 A)$. The results of MTT and transwell assays showed that the transfection of miR-199a inhibitor promoted the proliferation of breast cancer cells compared with the control group, and DMED promoted the proliferation of breast cancer cells compared with the miR199a inhibitor group $(\mathrm{P}<0.05$, Figure 3B,3C). On the other hand, the results of flow cytometry and caspase- 3 activity assay showed that, compared with the control group, the transfection of miR-199a inhibitor reduced the apoptosis rate and the activity of caspase- 3 in MCF-7 and MDAMB-231 cells. Compared with the miR-199a inhibitor group, DMED decreased the apoptosis of breast cancer cells $(\mathrm{P}<0.05$, Figure $3 D, 3 E)$. This suggests that DMED further enhanced the downregulation of miR-199a to promote the activity of breast cancer cells and inhibit apoptosis.

\section{MiR-199a targeted HIF-1a}

Subsequently, we explored the mechanism of miR-199a in breast cancer, screened the downstream targets of miR199a through bioinformation database and showed that HIF- $1 \alpha$ contained a conservative binding site of miR-199a (Figure 4A). For HIF-1 $\alpha$ wild type reporter gene plasmid, the luciferase activity of miR-199a mimic transfection group was significantly lower than that of the negative 

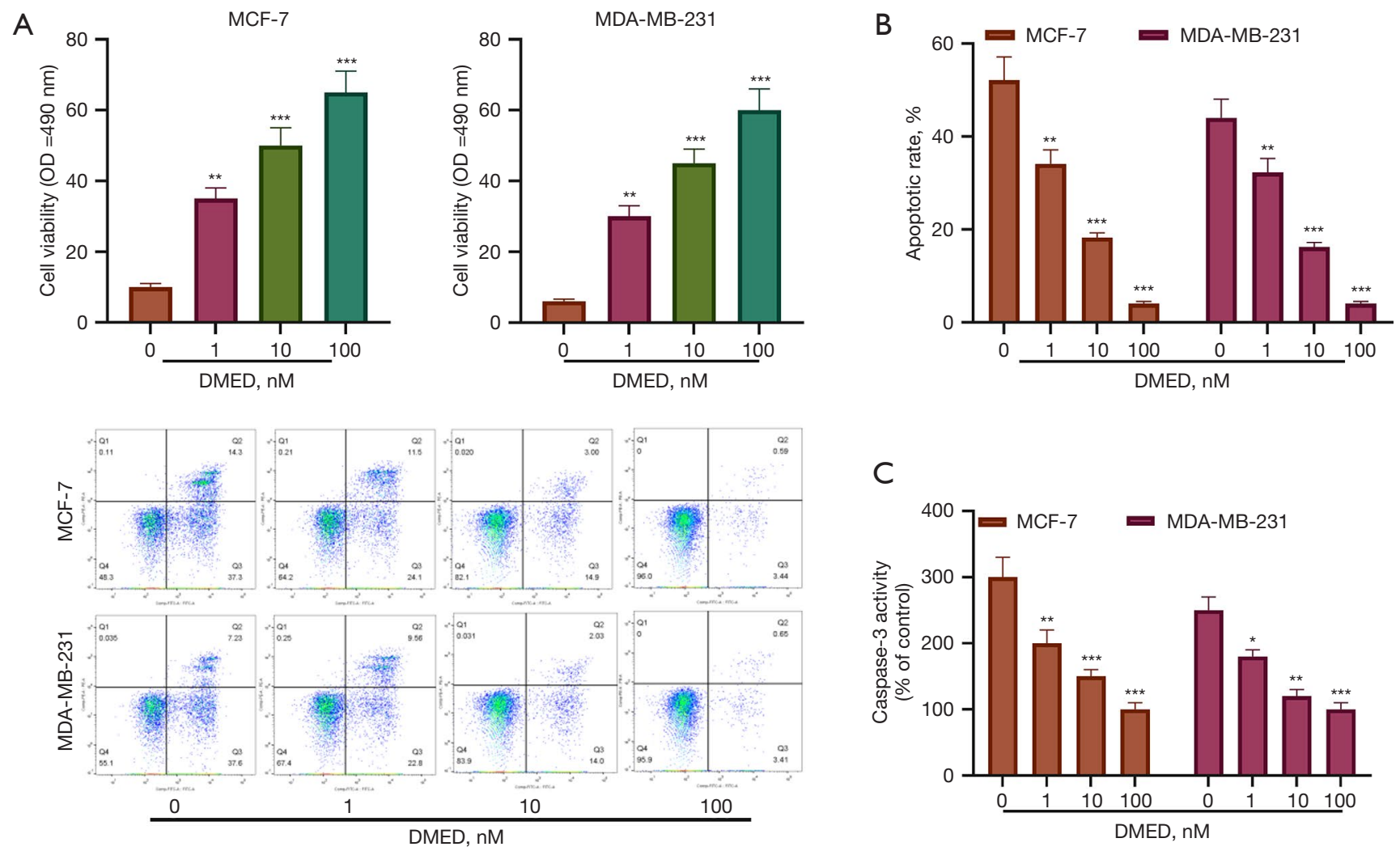

Figure 1 DMED induces cytotoxicity and apoptosis of breast cancer cells. MCF-7 and MDA-MB-231 cells were treated with different concentrations of DMED. (A) The activity of breast cancer cells was detected using the MTT assay. (B) Flow cytometry was used to detect apoptosis. (C) The level of apoptosis was detected using caspase-3 activity. * $\mathrm{P}<0.05 ;{ }^{* *}, \mathrm{P}<0.01 ;{ }^{* * *}, \mathrm{P}<0.001$. DMED, Dexmetomidine; MTT, 3-(4,5-dimethylthiazol-2-yl)-2,5-diphenyl-2H-tetrazolium bromide.

control group $(\mathrm{P}<0.05)$. For HIF-1 $\alpha$ mutant reporter gene plasmid, there was no significant difference in luciferase activity between the two groups $(\mathrm{P}>0.05$, Figure $4 B)$. Then, we explored the effect of DMED on the level of HIF-1 $\alpha$ in breast cancer cells. Results from QRT-PCR showed that DMED upregulated the level of HIF-1 $\alpha$ mRNA in MCF7 and MDA-MB-231 cells in a dose-dependent manner $(\mathrm{P}<0.05$, Figure $4 C)$. On the other hand, the transfection of miR-199a mimics and inhibitors downregulated and upregulated the expression of HIF- $1 \alpha$ in MCF-7 and MDAMB-231 cells, respectively $(\mathrm{P}<0.05$, Figure $4 D)$. These results indicate that HIF- $1 \alpha$ is the target of miR-199a and is negatively regulated by miR-199a.

\section{DMED promoted the progression of breast cancer through miR-199a/HIF-1a}

Finally, the mechanism of DMED regulating miR-199a/
HIF- $1 \alpha$ axis to inhibit the progression of breast cancer was explored. The results of QRT-PCR showed that, compared with the control group, the expression of HIF-1 $\alpha$ in DMED group was upregulated, the expression of HIF$1 \alpha$ in the HIF- $1 \alpha$ overexpression group was upregulated, and compared with the HIF- $1 \alpha$ overexpression group, the expression of HIF- $1 \alpha$ in the HIF- $1 \alpha+$ miR-199a mimics group was downregulated $(\mathrm{P}<0.05$, Figure $5 A)$. At the same time, the results of MTT and a transwell assay showed that, compared with the control group, DMED promoted the proliferation of breast cancer cells. Similarly, the overexpression of HIF-1 $\alpha$ promoted the proliferation of breast cancer cells. Compared with the HIF-1 $\alpha$ overexpression group, the cell activity of HIF- $1 \alpha+$ miR-199a mimics group was downregulated $(\mathrm{P}<0.05$, Figure $5 B, 5 C)$. Subsequently, the results of an apoptosis assay showed that DMED inhibited the apoptosis of breast cancer cells, and HIF- $1 \alpha$ also inhibited the apoptosis of breast cancer cells. 
A

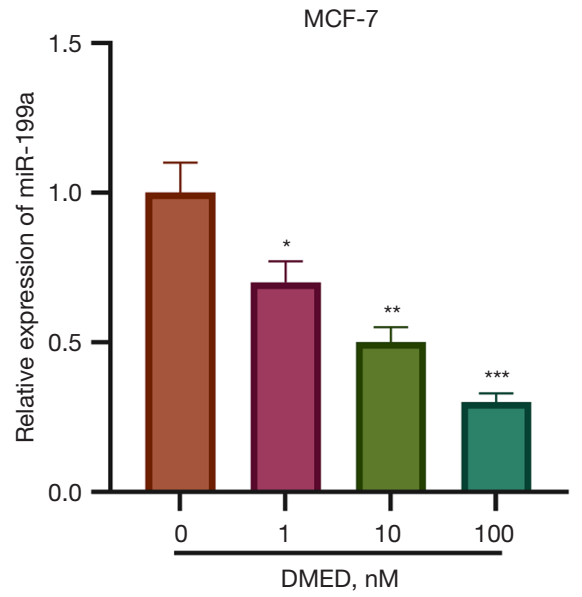

C

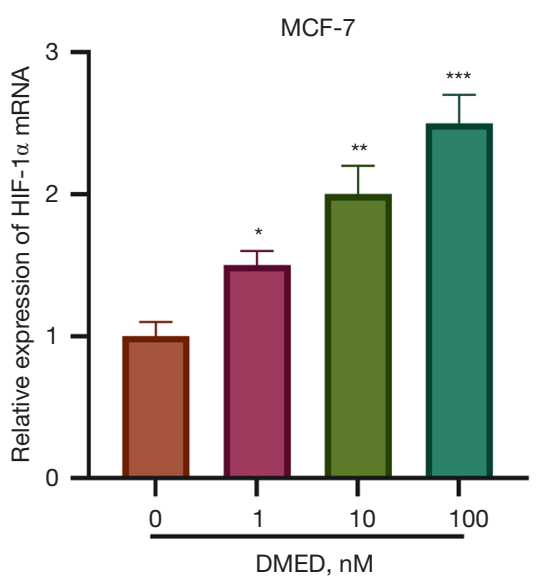

B
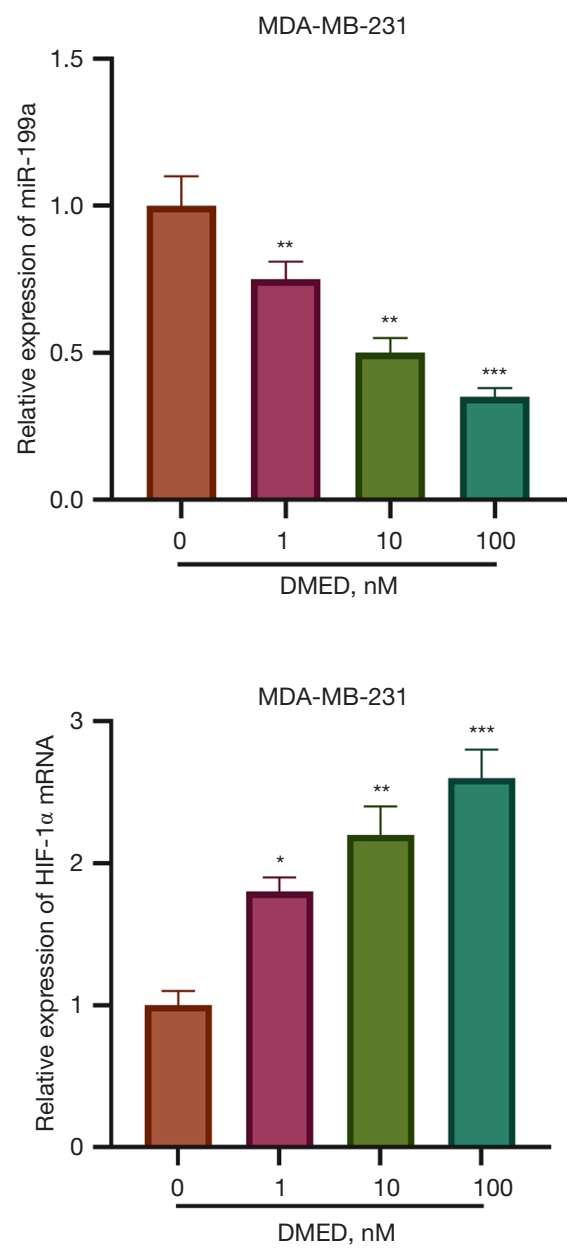

Figure 2 DMED downregulated the expression of miR-199a. MCF-7 and MDA-MB-231 cells were treated with different concentrations of DMED. (A,B) QRT-PCR was used to detect the level of miR-199a in cells. (C,D) QRT-PCR was used to detect the level of HIF-1 $\alpha$ in breast cancer cells. *, $\mathrm{P}<0.05$; ${ }^{* *}, \mathrm{P}<0.01$; ${ }^{* *}, \mathrm{P}<0.001$. DMED, Dexmetomidine; QRT-PCR, quantitative real-time polymerase chain reaction.

Compared with the vector group, on the other hand, and compared with the HIF- $1 \alpha$ overexpression group, apoptosis increased in the HIF-1 $\alpha+$ miR-199a mimics group $(\mathrm{P}<0.05$, Figure $5 D, 5 E)$. These results suggest that DMED regulates the miR-199a/HIF-1 $\alpha$ axis to promote the proliferation of breast cancer cells and inhibit apoptosis, thus promoting the progression of breast cancer.

\section{Discussion}

In recent years, great progress has been made in the clinical treatment of breast cancer, but the incidence of, and mortality from, breast cancer are still on the rise (23). Therefore, it is of great significance to find effective and safe anticancer drugs.
In addition, the study of the molecular mechanisms of breast cancer activity and apoptosis can provide a new basis for the treatment of breast cancer (24). It is of great significance to explore the molecular mechanism of drugs in the treatment of breast cancer.

In recent years studies have shown that DMED plays an important role in cell apoptosis and activity. Li et al. confirmed that DMED can significantly inhibit the loss of pulmonary microvascular endothelial cell (PMVEC) activity and apoptosis induced by IR serum (25). In addition, Sun et al. have confirmed that DMED can protect glial cells by reducing cell death (26). On the other hand, DMED is involved in the progression of cancer. Studies have shown that DEX can reduce the 

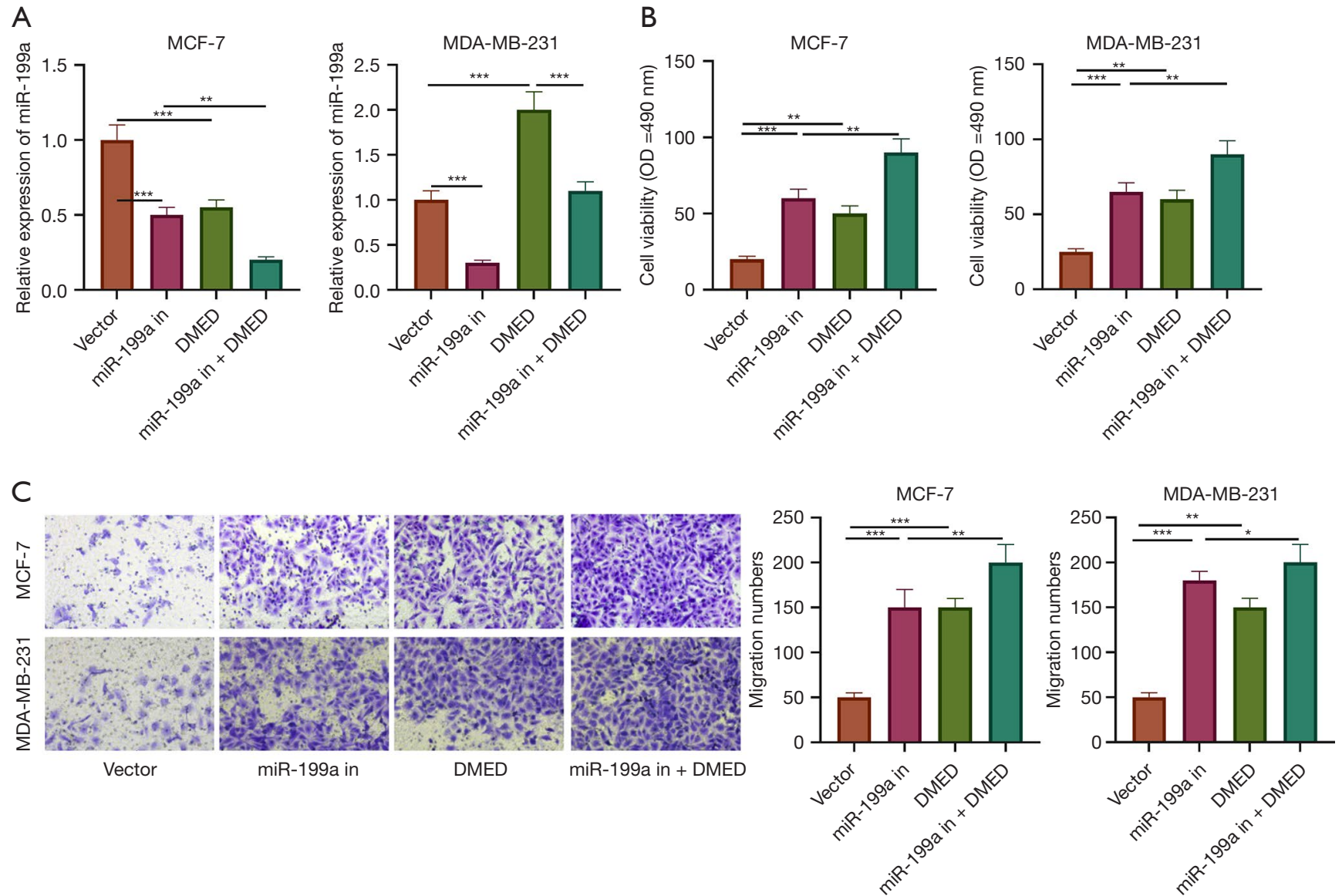

D
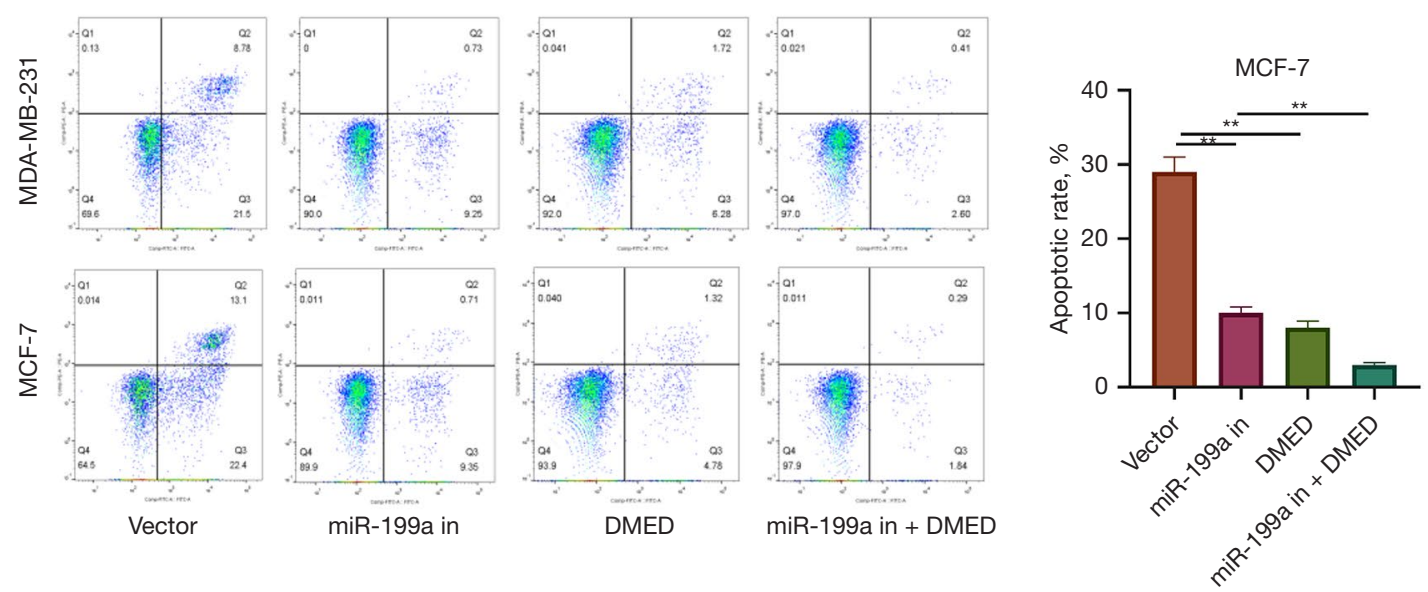

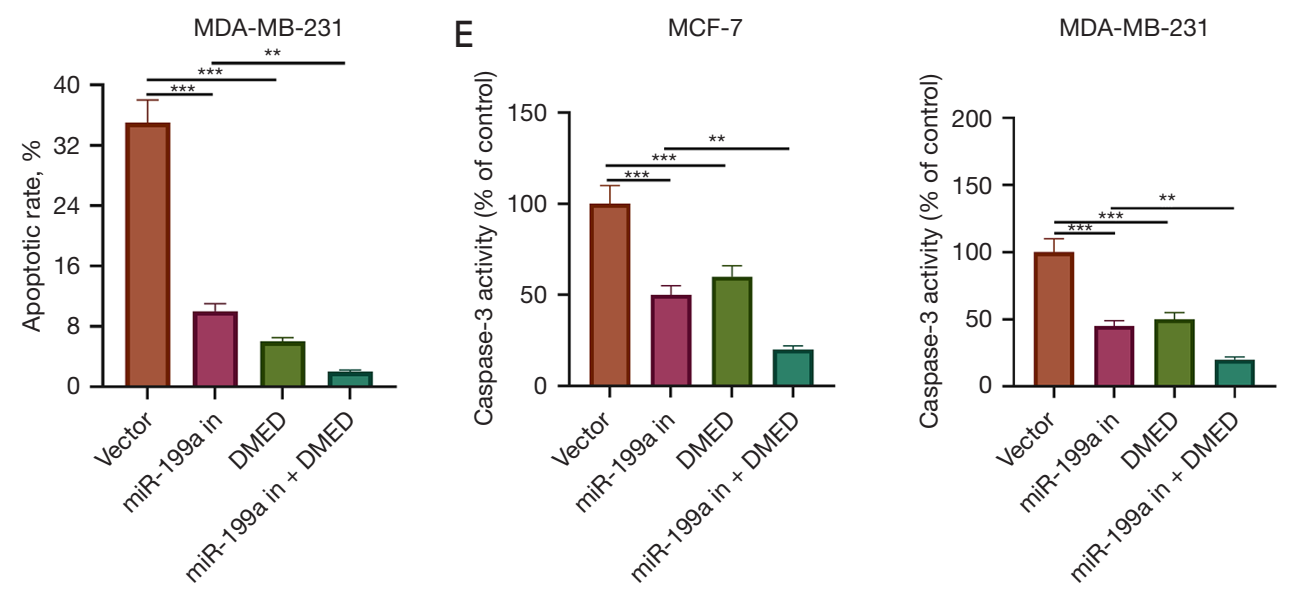

Figure 3 DMED can increase the downregulation of miR-199a expression, promote the activity of breast cancer cells and inhibit apoptosis. MCF-7 and MDA-MB-231 cells were transfected with miR-199a inhibitor. (A) QRT-PCR was used to detect the level of miR-199a in breast cancer cells. (B,C) MTT and transwell assays were used to detect cell proliferation (the transwell assay was stained with crystal violet with a magnification of $100 \times)$, and $(\mathrm{D}, \mathrm{E})$ flow cytometry and caspase-3 activity assay were used to detect cell apoptosis. *, $\mathrm{P}<0.05 ;{ }^{* *}, \mathrm{P}<0.01,{ }^{* * *}$, $\mathrm{P}<0.001$. DMED, Dexmetomidine; QRT-PCR, quantitative real-time polymerase chain reaction; MTT, 3-(4,5-dimethylthiazol-2-yl)-2,5diphenyl-2H-tetrazolium bromide.

A

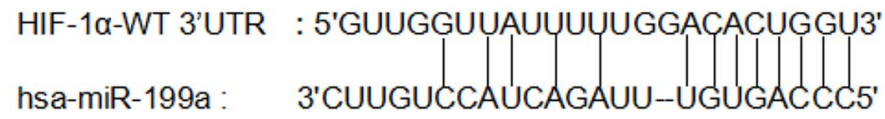

HIF-1 $\alpha$-MUT 3'UTR : 5'GUUGGUUAUUUUUGGACACUGGU3'

B

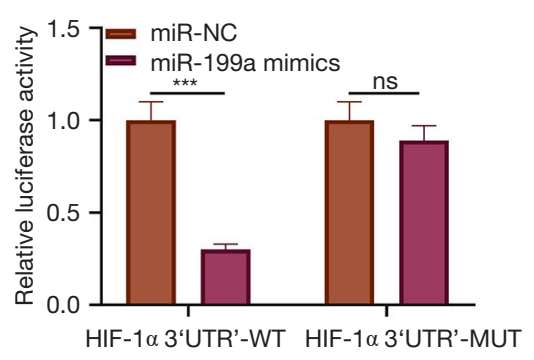

D

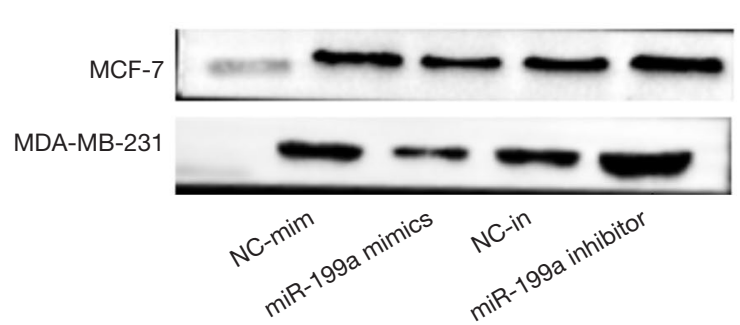

C
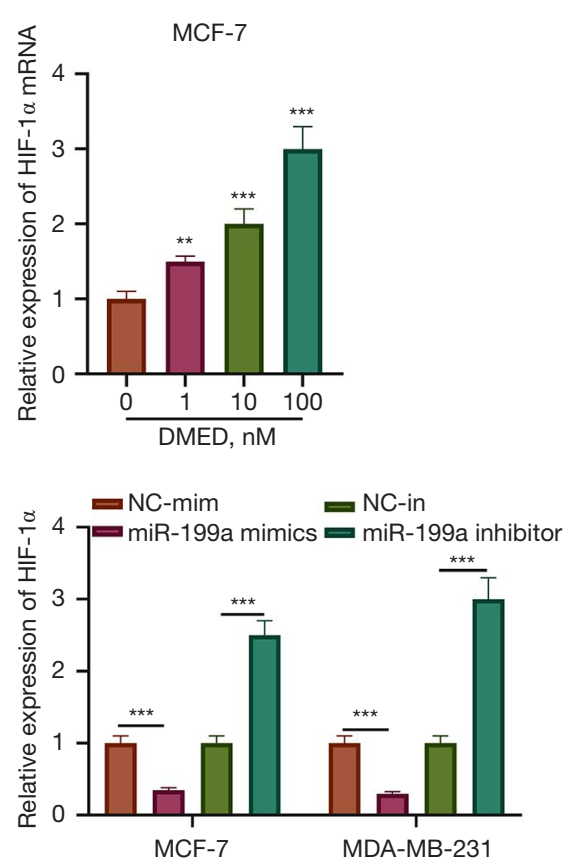

Figure 4 MiR-199a targeting HIF-1 $\alpha$. (A) Bioinformatics website shows that HIF-1 $\alpha$ contains miR-199a binding site. (B) Double luciferase assay was used to detect the binding of miR-199a and HIF-1 $\alpha$. (C) The level of HIF-1 $\alpha$ mRNA was detected using QRT-PCR. (D) The level of HIF-1 $\alpha$ was detected using western blot. ${ }^{* *}, \mathrm{P}<0.01{ }^{* * *}, \mathrm{P}<0.001$. QRT-PCR, quantitative real-time polymerase chain reaction. 

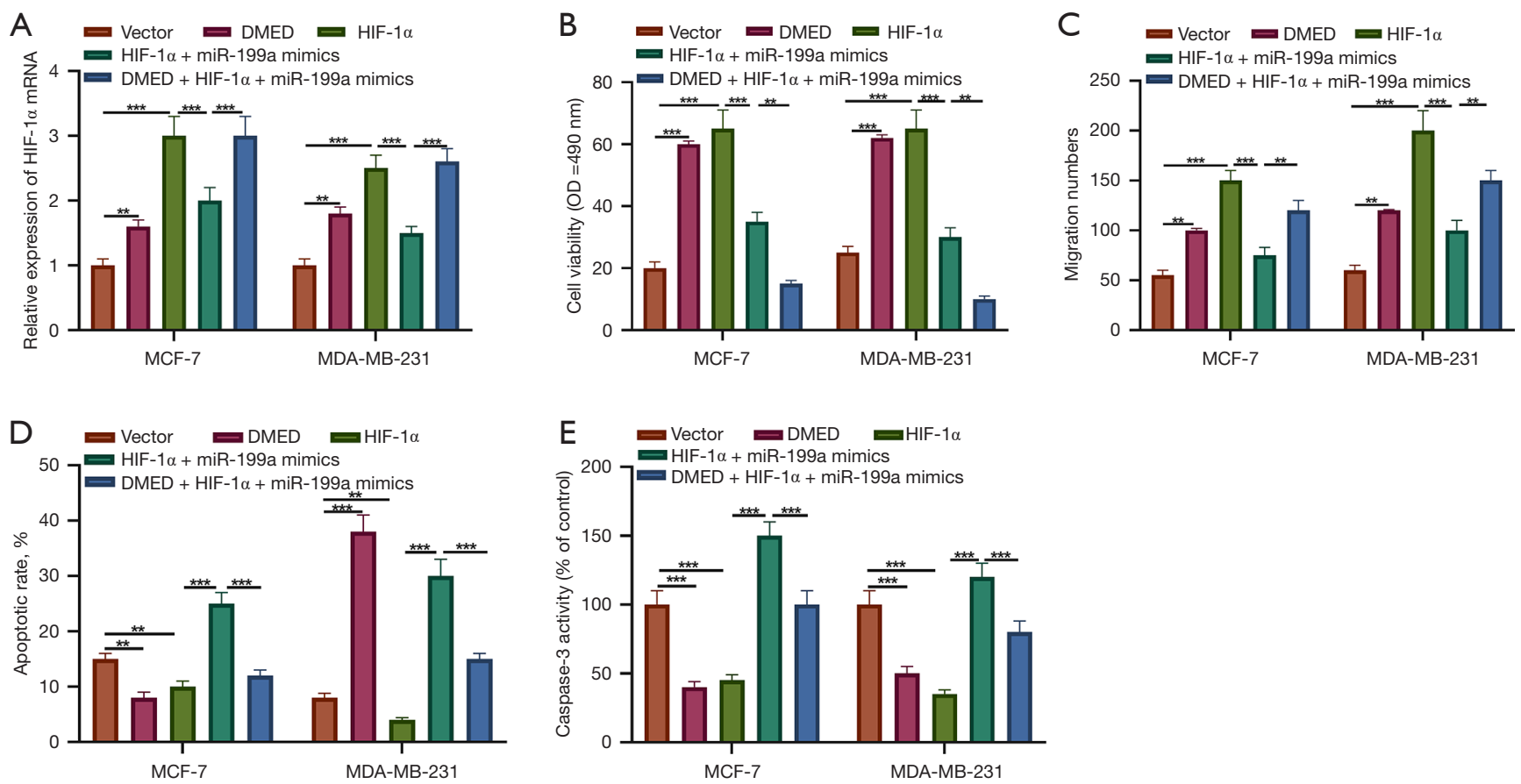

Figure 5 DMED promotes the progression of breast cancer through the miR-199a/HIF-1 $\alpha$ axis. HIF-1 $\alpha$ overexpression plasmid and miR199a mimics were used to co-transfect MCF-7 and MDA-MB-231 cells. (A) The level of HIF-1 $\alpha$ mRNA in breast cancer cells was detected using QRT-PCR. (B,C) MTT and transwell assays were used to detect cell proliferation. (D,E) Flow cytometry and caspase-3 activity assay were used to detect cell apoptosis. ${ }^{* *}, \mathrm{P}<0.01$; ${ }^{* * *}, \mathrm{P}<0.001$. DMED, Dexmetomidine; QRT-PCR, quantitative real-time polymerase chain reaction; MTT, 3-(4,5-dimethylthiazol-2-yl)-2,5-diphenyl-2H-tetrazolium bromide.

incidence and intensity of delirium after pneumonectomy in elderly patients with lung cancer. At the same time, DMED can provide effective cardio-brain protection in colorectal cancer surgery (27). Recent animal data suggest that DMED has a potential role in promoting cancer recurrence and metastasis during the perioperative period, especially after breast surgery (28). Other studies have shown that DMED has a protective effect on disease by regulating miRNA. Studies by Bao et al. showed that DMED may play an anti-inflammatory effect in LPS-stimulated BV2 cells by upregulating miR-340, and DMED may be a potential drug for the treatment of neuroinflammation (29). Similarly, miRNA-151-3p enhanced the neuroprotective effect of DMED on A $\beta$ by targeting DAPK-1 and TP53 (30). In esophageal cancer, DMED inhibits the progression of esophageal cancer through the miR-143-3p/EPS8 axis (10). In addition, DMED upregulates miRNA-185 by inhibiting the SOX9/ $\mathrm{Wnt} / \beta$-catenin signaling pathway to inhibit ovarian cancer growth (31). In this study, we found that DMED can downregulate the expression of miR-199a in breast cancer
MCF-7 and MDA-MB-231 cells. Further experiments showed that DMED promoted the activity of breast cancer cells and inhibited apoptosis through the miR-199a/HIF$1 \alpha$ axis.

MiR-199a plays an important role in regulating cell survival, apoptosis, and tumor cell metastasis. For example, miR-199a can inhibit the activity of thyroid cancer cells and promote apoptosis (32). MiRNA is associated with breast cancer cell activity and apoptosis. For example, miRNA-106a promotes breast cancer cell proliferation, clone formation, migration, and invasion by inhibiting apoptosis (33). In addition, studies by $\mathrm{Xu} e t \mathrm{al}$. showed that miR-199a can inhibit the activity of breast cancer (34). MiR-199a is regulated by drugs. Guo et al. confirmed that quercetin downregulates the viability of miR-199a Sertoli cells and inhibits cardiomyocyte apoptosis (35). Similarly, propofol induces apoptosis in hepatocellular carcinoma cells by upregulating the expression of miRNA-199a (36). On the other hand, miR-199a can play a role in cancer or breast cancer by targeting mRNA, and studies by Ma et al. confirmed that miR-199a inhibits the progression 
of papillary thyroid cancer by targeting SNAI1 (37). In breast cancer, miR-199a regulates MRP1 to inhibit drug resistance in breast cancer cells (16). In breast cancer, miR$29 \mathrm{~b}-3 \mathrm{p}$ promotes the activity of breast cancer cells by downregulating TRAF3 (38). HIF- $1 \alpha$ is a transcriptional regulatory factor that exists widely in mammalian and human cells. Studies have confirmed that HIF-1 $\alpha$ is highly expressed in many human tumors, which is closely related to tumor growth, reproduction, invasion and metastasis, neovascularization, apoptosis (39). On the other hand, Cao et al. have confirmed that HIF- $1 \alpha$ can promote the activity of breast cancer cells (19). Some studies have also confirmed that HIF- $1 \alpha$ is regulated by miRNA in cancer. Wu et al. confirmed that miR-49 inhibits angiogenesis in breast cancer by targeting HIF-1 $\alpha$ (40). Previous studies confirmed that miR-199a protects PC12 cells from oxidative damage by regulating HIF-1 $\alpha$ (17). In this study, it was found that downregulation of miR-199a expression could upregulate the expression of HIF-1 $\alpha$, and DMED could upregulate the expression of HIF-1 $\alpha$. Further experiments confirmed that HIF-1 $\alpha$ was the direct target of miR-199a and could partially reverse the inhibitory effect of miR-199a on apoptosis of breast cancer cells. Therefore, we speculate that in breast cancer, miR-199a can target HIF-1 $\alpha$ and inhibit the progression of breast cancer.

\section{Conclusions}

This study found that DMED could inhibit the progression of MCF-7 and MDA-MB-231 cells by regulating the miR199a/HIF-1 $\alpha$ axis. However, this study has shortcomings. Animal experiments need to further explore the role of DMED regulating miR-199a in the activity of breast cancer cells. In addition, it needs to be assessed whether DMED can regulate other miRNAs to play a role in the activity of breast cancer cells. In short, the findings of this study can provide guidance for the treatment of DMED and molecular targeted therapy in breast cancer.

\section{Acknowledgments}

Funding: None.

\section{Footnote}

Reporting Checklist: The authors have completed the MDAR reporting checklist. Available at https://dx.doi. org/10.21037/tcr-21-1937
Data Sharing Statement: Available at https://dx.doi. org/10.21037/tcr-21-1937

Conflicts of Interest: Both authors have completed the ICMJE uniform disclosure form (available at https://dx.doi. org/10.21037/tcr-21-1937). Both authors have no conflicts of interest to declare.

Ethical Statement: The authors are accountable for all aspects of the work in ensuring that questions related to the accuracy or integrity of any part of the work are appropriately investigated and resolved.

Open Access Statement: This is an Open Access article distributed in accordance with the Creative Commons Attribution-NonCommercial-NoDerivs 4.0 International License (CC BY-NC-ND 4.0), which permits the noncommercial replication and distribution of the article with the strict proviso that no changes or edits are made and the original work is properly cited (including links to both the formal publication through the relevant DOI and the license). See: https://creativecommons.org/licenses/by-nc-nd/4.0/.

\section{References}

1. Coker-Gurkan A, Can E, Sahin S, et al. Atiprimod triggered apoptotic cell death via acting on PERK/eIF2 $\alpha /$ ATF4/CHOP and STAT3/NF-KB axis in MDA-MB-231 and MDA-MB-468 breast cancer cells. Mol Biol Rep 2021;48:5233-47.

2. Depboylu B. Treatment and patient related quality of life issues in elderly and very el-derly breast cancer patients. Transl Cancer Res 2020;9:S146-53.

3. Pellicciaro M, Materazzo M, Buonomo C, et al. Feasibility and oncological safety of axillary reverse mapping in patients with locally advanced breast cancer and partial response after neoadjuvant chemotherapy. In Vivo 2021;35:2489-94.

4. Zhou Y, Yue Y, Fan S, et al. Advances in pathophysiology of triple-negative breast cancer: the potential of $\operatorname{lncRNAs}$ for clinical diagnosis, treatment, and prognostic monitoring. Mol Biotechnol 2021. [Epub ahead of print]. doi: 10.1007/s12033-021-00368-x.

5. Ezzati M, Yousefi B, Velaei K, et al. A review on anticancer properties of Quercetin in breast cancer. Life Sci 2020;248:117463.

6. Qiu J, Zhang T, Zhu X, et al. Hyperoside induces breast cancer cells apoptosis via ROS-mediated NF- $\mathrm{\kappa B}$ signaling 
pathway. Int J Mol Sci 2019;21:131.

7. Keating GM. Dexmedetomidine: A Review of Its Use for Sedation in the Intensive Care Setting. Drugs 2015;75:1119-30.

8. Zhai M, Liu C, Li Y, et al. Dexmedetomidine inhibits neuronal apoptosis by inducing Sigma-1 receptor signaling in cerebral ischemia-reperfusion injury. Aging (Albany NY) 2019;11:9556-68.

9. Lavon H, Matzner P, Benbenishty A, et al. Dexmedetomidine promotes metastasis in rodent models of breast, lung, and colon cancers. Br J Anaesth 2018;120:188-96.

10. Zhang P, He H, Bai Y, et al. Dexmedetomidine suppresses the progression of esophageal cancer via miR-143-3p/ epidermal growth factor receptor pathway substrate 8 axis. Anticancer Drugs 2020;31:693-701.

11. Chi M, Shi X, Huo X, et al. Dexmedetomidine promotes breast cancer cell migration through Rab11-mediated secretion of exosomal TMPRSS2. Ann Transl Med 2020;8:531.

12. Xia M, Ji NN, Duan ML, et al. Dexmedetomidine regulate the malignancy of breast cancer cells by activating a2-adrenoceptor/ERK signaling pathway. Eur Rev Med Pharmacol Sci 2016;20:3500-6.

13. Debnath T, Deb Nath NC, Kim EK, et al. Role of phytochemicals in the modulation of miRNA expression in cancer. Food Funct 2017;8:3432-42.

14. Fridrichova I, Zmetakova I. MicroRNAs Contribute to Breast Cancer Invasiveness. Cells 2019;8:1361.

15. Hu D, Li M, Su J, et al. Dual-targeting of miR-124$3 \mathrm{p}$ and $\mathrm{ABCC} 4$ promotes sensitivity to Adriamycin in breast cancer cells. Genet Test Mol Biomarkers 2019;23:156-65.

16. Chang L, Hu Z, Zhou Z, et al. Linc00518 contributes to multidrug resistance through regulating the miR199a/MRP1 axis in breast cancer. Cell Physiol Biochem 2018;48:16-28.

17. Wu L, Xi Y, Kong Q. Dexmedetomidine protects PC12 cells from oxidative damage through regulation of miR-199a/HIF-1 $\alpha$. Artif Cells Nanomed Biotechnol 2020;48:506-14.

18. Zhu Y, Zhao H, Zhang W, et al. Dexmedetomidine attenuates neuronal injury induced by cerebral ischemia-reperfusion by regulating miR-199a. Mol Med Rep 2021;24:574.

19. Cao L, Wang M, Dong Y, et al. Circular RNA circRNF20 promotes breast cancer tumorigenesis and Warburg effect through miR-487a/HIF-1 $\alpha / \mathrm{HK} 2$. Cell Death Dis
2020;11:145.

20. Zhao W, Geng D, Li S, et al. LncRNA HOTAIR influences cell growth, migration, invasion, and apoptosis via the miR-20a-5p/HMGA2 axis in breast cancer. Cancer Med 2018;7:842-55.

21. Shi XY, Wang H, Wang W, et al. MiR-98-5p regulates proliferation and metastasis of MCF-7 breast cancer cells by targeting Gab2. Eur Rev Med Pharmacol Sci 2020;24:10914.

22. Choudhary GS, Al-Harbi S, Almasan A. Caspase-3 activation is a critical determinant of genotoxic stressinduced apoptosis. Methods Mol Biol 2015;1219:1-9.

23. Sopik V. International variation in breast cancer incidence and mortality in young women. Breast Cancer Res Treat 2021;186:497-507.

24. González-González A, Mediavilla MD, Sánchez-Barceló EJ. Melatonin: a molecule for reducing breast cancer risk. Molecules 2018;23:336.

25. Li J, Chen Q, He X, et al. Dexmedetomidine attenuates lung apoptosis induced by renal ischemia-reperfusion injury through $\alpha 2 \mathrm{AR} / \mathrm{PI} 3 \mathrm{~K} / \mathrm{Akt}$ pathway. J Transl Med 2018;16:78.

26. Sun YB, Zhao H, Mu DL, et al. Dexmedetomidine inhibits astrocyte pyroptosis and subsequently protects the brain in in vitro and in vivo models of sepsis. Cell Death Dis 2019;10:167.

27. Yi XL, Wang JT, Chu CQ, et al. Cardiocerebral protective effects of dexmedetomidine as anesthetic in colorectal cancer surgery. Eur Rev Med Pharmacol Sci 2018;22:3570-6.

28. Nair AS, Saifuddin MS, Naik V, et al. Dexmedetomidine in cancer surgeries: Present status and consequences with its use. Indian J Cancer 2020;57:234-8.

29. Bao Y, Zhu Y, He G, et al. Dexmedetomidine attenuates neuroinflammation in LPS-stimulated BV2 microglia cells through upregulation of miR-340. Drug Des Devel Ther 2019;13:3465-75.

30. Guo Y, Wu Y, Li N, et al. Up-regulation of miRNA151-3p enhanced the neuroprotective effect of dexmedetomidine against $\beta$-amyloid by targeting DAPK-1 and TP53. Exp Mol Pathol 2021;118:104587.

31. Tian H, Hou L, Xiong Y, et al. Dexmedetomidine upregulates microRNA-185 to suppress ovarian cancer growth via inhibiting the SOX9/Wnt/ $\beta$-catenin signaling pathway. Cell Cycle 2021;20:765-80.

32. Wang D, Zheng Y. Silencing long non-coding RNAs nicotinamide nucleotide transhydrogenase antisense RNA 1 inhibited papillary thyroid cancer cell proliferation, 
migration and invasion and promoted apoptosis via targeting miR-199a-5p. Endocr J 2021;68:583-97.

33. You F, Luan H, Sun D, et al. miRNA-106a promotes breast cancer cell proliferation, clonogenicity, migration, and invasion through inhibiting apoptosis and chemosensitivity. DNA Cell Biol 2019;38:198-207.

34. Xu ST, Xu JH, Zheng ZR, et al. Long non-coding RNA ANRIL promotes carcinogenesis via sponging miR-199a in triple-negative breast cancer. Biomed Pharmacother 2017;96:14-21.

35. Guo G, Gong L, Sun L, et al. Quercetin supports cell viability and inhibits apoptosis in cardiocytes by downregulating miR-199a. Artif Cells Nanomed Biotechnol 2019;47:2909-16.

36. Zhang J, Wu GQ, Zhang Y, et al. Propofol induces apoptosis of hepatocellular carcinoma cells by upregulation of microRNA-199a expression. Cell Biol

Cite this article as: Wen G, Xin N. Dexmetomidine promotes the activity of breast cancer cells through miR-199a/HIF-1 $\alpha$ axis. Transl Cancer Res 2021;10(11):4817-4828. doi: 10.21037/tcr21-1937
Int 2013;37:227-32.

37. Ma S, Jia W, Ni S. miR-199a-5p inhibits the progression of papillary thyroid carcinoma by targeting SNAI1. Biochem Biophys Res Commun 2018;497:181-6.

38. Zhang B, Shetti D, Fan C, et al. miR-29b-3p promotes progression of MDA-MB-231 triple-negative breast cancer cells through downregulating TRAF3. Biol Res 2019;52:38.

39. Wei TT, Lin YT, Tang SP, et al. Metabolic targeting of $\mathrm{HIF}-1 \alpha$ potentiates the therapeutic efficacy of oxaliplatin in colorectal cancer. Oncogene 2020;39:414-27.

40. Wu Z, Cai X, Huang C, et al. miR-497 suppresses angiogenesis in breast carcinoma by targeting HIF-1 $\alpha$. Oncol Rep 2016;35:1696-702.

(English Language Editor: B. Meiser) 